\title{
Interventionen zur Vorbeugung von Gestationsdiabetes bei Frauen: eine Übersicht über Cochrane Reviews
}

Griffith R], Alsweiler ], Moore AE, Brown S, Middleton P, Shepherd E, Crowther CA. Interventions to prevent women from developing gestational diabetes mellitus: an overview of Cochrane Reviews. Cochrane Database of Systematic Reviews 2020, Issue 6. Art. No.: CD012394. DOI: 10.1002/14651858.CD012394. pub3.

\section{Worum geht es?}

Der Gestationsdiabetes (GDM, Schwangerschaftsdiabetes) ist definiert als ein hoher Blutzuckerspiegel (Hyperglykämie), der erstmals während der Schwangerschaft festgestellt wird. Wenn die Mutter einen GDM entwickelt, kann die Gesundheit von Frauen und ihren Kindern beeinträchtigt werden.

Während der Schwangerschaft verändert der Körper einer Frau die Art und Weise, wie er die Nährstoffe aus ihrer Nahrung verarbeitet, um sicherzustellen, dass das Kind gut ernährt wird. In den ersten drei Monaten hat die Mutter eine erhöhte Insulinsensitivität. Im zweiten und dritten Trimester ist ihre Insulinsensitivität vermindert. Bei Frauen mit GDM ist der anfängliche Anstieg der Sensitivität geringer, und ihre Insulinsensitivität ist später in der Schwangerschaft über das normale Maß hinaus vermindert, was dazu führt, dass die Mutter hohe Blutzuckerwerte entwickelt. Ihre Blutfettwerte sind ebenfalls höher als normal, was zum Risiko beitragen kann, dass das Baby für sein Gestationsalter (die Schwangerschaftsdauer) groß wird.

\section{Warum ist das wichtig?}

Frauen mit GDM entwickeln häufiger Komplikationen in der Schwangerschaft, einschließlich Bluthochdruck und der Notwendigkeit einer Geburtseinleitung. Sie sind später einem erhöhten Risiko für die Entwicklung eines Typ-2-Diabetes ausgesetzt. Kinder, die von Frauen mit GDM geboren werden, werden mit größerer Wahrscheinlichkeit groß geboren und erleiden daher häufiger Geburtsverletzungen. Nach der Geburt haben die Säuglinge ein höheres Risiko für Atembeschwerden, eine Gelbsucht und einen verringerten Blutzuckerspiegel, und später für Fettleibigkeit und Diabetes.

Es gibt viele Risikofaktoren für GDM, wodurch es wahrscheinlich ist, dass Interventionen vor oder während der Schwangerschaft das Risiko von Frauen, GDM zu entwickeln, verringern könnten. Diese Übersicht fasst die Evidenz aus Cochrane Reviews mit randomisierten kontrollierten Studien zu Interventionen zusammen, die GDM verhindern könnten.

\section{Welche Evidenz haben wir gefunden?}

Wir durchsuchten die Cochrane Library im August 2019. Wir identifizierten 11 Cochrane Reviews, die Interventionen während der Schwangerschaft bewerteten und über GDM berichteten. Die Reviews enthielten Ergebnisse aus 71 randomisierten kontrollierten Studien mit 23.154 schwangeren Frauen. Die Interventionen umfassten Diät, Bewegung, eine Kombination aus Diät und Bewegung, Nahrungsergänzungsmittel, Medikamente und die Behandlung anderer Gesundheitsprobleme. Die Qualität der Evidenz reichte von sehr niedrig bis hoch. Wir fanden 10 weitere Cochrane Reviews, die künftig mehr Informationen zu diesem Thema liefern könnten.

\section{Ernährung und Bewegung}

Ernährung und körperliche Bewegung zusammen verringerten möglicherweise das Risiko einer Frau, GDM zu entwickeln, im Vergleich zur Standardbehandlung (19 Studien; 6633 Frauen; moderate Qualität der Evidenz). 
Ernährungsberatung allein hatte eine unklare Wirkung auf das Risiko für GDM (5 Studien; 1279 Frauen; sehr niedrige Qualität der Evidenz). Eine Diät mit niedrigem glykämischen Index im Vergleich zu einer Diät mit mittlerem bis hohem glykämischen Index hatte ebenfalls eine unklare Wirkung (4 Studien; 912 Frauen; niedrige Qualität der Evidenz). Bewegung allein hatte eine unklare Wirkung auf das Risiko (3 Studien; 826 Frauen; niedrige Qualität der Evidenz).

\section{Nahrungsergänzungsmittel}

Eine Supplementierung mit Omega-3Fettsäuren in der Schwangerschaft hatte keine Wirkung (12 Studien; 5235 Frauen; hohe Qualität der Evidenz).

Eine Supplementierung mit Myo-Inositol während der Schwangerschaft verringerte möglicherweise das Risiko für GDM (3 Studien mit 502 Frauen; niedrige Qualität der Evidenz).

Eine Vitamin-D-Supplementierung in der Schwangerschaft hatte möglicherweise einen Nutzen bezogen auf die Verringerung des Risikos der Entwicklung von GDM (4 Studien mit 446 Frauen; niedrige Qualität der Evidenz). Diese Studien stammten alle aus asiatischen Ländern, und der Vitamin-
D-Spiegel der Frauen vor der Supplementierung war weitgehend unbekannt.

Vitamin D, das mit Nahrungsergänzungsmitteln wie Kalzium oder mit Kalzium plus anderen Mineralien verabreicht wurde, hatte eine unklare Wirkung.

Probiotika mit diätetischer Intervention hatten eine unklare Wirkung auf das Risiko der Entwicklung von GDM.

\section{Medikamente}

Das Medikament Metformin zeigte einen möglichen Nutzen bei der Verringerung des Risikos der Entwicklung von GDM bei der Verabreichung an adipöse Schwangere (3 Studien; 892 Frauen; moderate Qualität der Evidenz).

Evidenz von niedriger bis sehr niedriger Qualität aus acht kleinen Studien zeigte eine unklare Wirkung auf das GDM-Risiko für Heparin, Aspirin, Leukozyten-Immunisierung oder Immunglobulin (IgG) bei Frauen, die zuvor eine Totgeburt erlebt hatten.

\section{Management anderer Gesundheitsprobleme}

Ein universelles versus risikobasiertes Screening auf Schilddrüsenprobleme hatte keinen Einfluss auf das Risiko von GDM (1 Studie; 4516 Frauen; moderate Qualität der Evidenz). Zwei verschiedene Ansätze zur Behandlung von Asthma bei den Müttern hatten eine unklare Wirkung (niedrige Qualität der Evidenz).

\section{Was bedeutet das?}

Eine Kombination aus Bewegung und Ernährung, eine Supplementierung mit Myo-Inositol und eine Supplementierung mit Vitamin D waren von möglichem Nutzen bezogen auf die Verringerung des Risikos der Entwicklung von GDM. Um diese Ergebnisse zu bestätigen und den Einsatz von Metformin weiter zu untersuchen, ist weitere Evidenz von hoher Qualität aus randomisierten kontrollierten Studien erforderlich. In keiner der Studien wurden Interventionen vor der Schwangerschaft untersucht.

\section{Bibliografie}

Die Hebamme 2020; 33: 7-8

DOI 10.1055/a-1247-8612

ISSN 0932-8122

(c) 2020. Thieme. All rights reserved. Georg Thieme Verlag KG, Rüdigerstraße 14, 70469 Stuttgart, Germany' 